Göring, Frank; Harant, Jochen:

\title{
On domination in graphs
}

URN: $\quad$ urn:nbn:de:gbv:Ilm1-2020200110

\begin{tabular}{|c|c|}
\hline Original published in: & $\begin{array}{l}\text { Discussiones mathematicae. Graph theory / Uniwersytet Zielonogórski, } \\
\text { Wydział Matematyki, Informatyki i Ekonometrii. - Warsaw : De Gruyter } \\
\text { Open. - } 25 \text { (2005), 1/2, p. 7-12. }\end{array}$ \\
\hline Original published: & 2005 \\
\hline ISSN: & 2083-5892 \\
\hline DOI: & 10.7151/dmgt.1254 \\
\hline \multirow[t]{2}{*}{ [Visited: } & 2020-01-17] \\
\hline & $\begin{array}{l}\text { This work is licensed under a Creative Commons Attribution- } \\
\text { NonCommercial-NoDerivatives } 3.0 \text { Unported license. } \\
\text { To view a copy of this license, visit } \\
\text { http://creativecommons.org/licenses/BY-NC-ND/3.0/ }\end{array}$ \\
\hline
\end{tabular}


Discussiones Mathematicae

Graph Theory 25 (2005) 7-12

\title{
ON DOMINATION IN GRAPHS
}

\author{
FRANK GÖRING \\ Department of Mathematics \\ Chemnitz University of Technology \\ D-09107 Chemnitz, Germany \\ e-mail: frank.goering@mathematik.tu-chemnitz.de \\ AND \\ JOCHEN HARANT \\ Department of Mathematics \\ Technical University of Ilmenau \\ D-98684 Ilmenau, Germany \\ e-mail: harant@mathematik.tu-ilmenau.de
}

\begin{abstract}
For a finite undirected graph $G$ on $n$ vertices two continuous optimization problems taken over the $n$-dimensional cube are presented and it is proved that their optimum values equal the domination number $\gamma$ of $G$. An efficient approximation method is developed and known upper bounds on $\gamma$ are slightly improved.
\end{abstract}

Keywords: graph, domination.

2000 Mathematics Subject Classification: 05C69.

\section{Introduction and Results}

For terminology and notation not defined here we refer to [3]. Let $V=$ $V(G)=\{1, \ldots, n\}$ be the vertex set of an undirected graph $G$, and for $i \in V$, $N(i)$ be the neighbourhood of $i$ in $G, N_{2}(i)=\left\{k \in V \mid k \in \bigcup_{j \in N(i)} N(j) \backslash\right.$ $(\{i\} \cup N(i))\}, d_{i}=|N(i)|, t_{i}=\left|N_{2}(i)\right|, \delta=\min _{i \in V} d_{i}$, and $\Delta=\max _{i \in V} d_{i}$.

A set $D \subseteq V(G)$ is a dominating set of $G$ if $(\{i\} \cup N(i)) \cap D \neq \emptyset$ for every $i \in V$. The minimum cardinality of a dominating set of $G$ is the domination 
number $\gamma$ of $G$. In $[7] \gamma=\min _{x_{1}, \ldots, x_{n} \in[0,1]} \sum_{i \in V}\left(x_{i}+\left(1-x_{i}\right) \prod_{j \in N(i)}\left(1-x_{j}\right)\right)$ was proved. With $x_{1}=\ldots=x_{n}=x$ we have $\gamma \leq\left(x+(1-x)^{\delta+1}\right) n \leq$ $\left(x+e^{-(\delta+1) x}\right) n$ for every $x \in[0,1]$. Minimizing $x+(1-x)^{\delta+1}$ and $x+e^{-(\delta+1) x}$, the well-known inequalities $\gamma \leq\left(1-\frac{1}{(\delta+1)^{\frac{1}{\delta}}}+\frac{1}{(\delta+1)^{\frac{\delta+1}{\delta}}}\right) n \leq \frac{1+\ln (\delta+1)}{\delta+1} n$ (see $[4,8])$ follow. Obviously, it is easily checked whether $\gamma=1$ or not. Thus, we will assume $G \in \Gamma$ in the sequel, where $\Gamma$ is the set of graphs $G$ such that each component of $G$ has domination number greater than 1 . Without mentioning in each case, we will use $d_{i}, t_{i} \geq 1$ for $i=1, \ldots, n$ if $G \in \Gamma$. For $x_{1}, \ldots, x_{n} \in[0,1]$ let

$$
\begin{aligned}
f_{G}\left(x_{1}, \ldots, x_{n}\right)= & \sum_{i \in V}\left(x_{i}\left(1-\left(\prod_{j \in N(i)} x_{j}\right)\left(1-\prod_{k \in N_{2}(i)} x_{k}\right)\right)+\left(1-x_{i}\right) \prod_{j \in N(i)}\left(1-x_{j}\right)\right) \\
g_{G}\left(x_{1}, \ldots, x_{n}\right)= & f_{G}\left(x_{1}, \ldots, x_{n}\right) \\
& -\sum_{i \in V}\left(\frac{1}{1+d_{i}}\left(1-x_{i}\right)\left(\prod_{j \in N(i)}\left(1-x_{j}\right)\right)\left(\prod_{k \in N_{2}(i)}\left(1-x_{k}\right)\right)\right) .
\end{aligned}
$$

Theorem 1. If $G \in \Gamma$ then

$$
\begin{aligned}
\gamma & =\min _{x_{1}, \ldots, x_{n} \in[0,1]} f_{G}\left(x_{1}, \ldots, x_{n}\right)=\min _{x_{1}, \ldots, x_{n} \in[0,1]} g_{G}\left(x_{1}, \ldots, x_{n}\right) \\
& \leq \min _{x \in[0,1]} \sum_{i \in V}\left(x\left(1-x^{d_{i}}\left(1-x^{t_{i}}\right)\right)+(1-x)^{d_{i}+1}\left(1-\frac{1}{1+d_{i}}(1-x)^{t_{i}}\right)\right) \\
& \leq \min _{x \in[0,1]}\left(x\left(1-x^{\Delta}(1-x)\right)+(1-x)^{\delta+1}\left(1-\frac{1}{1+\Delta}(1-x)^{\Delta(\Delta-1)}\right)\right) n .
\end{aligned}
$$

Since DOMINATING SET is an NP-complete decision problem ([5]), it is difficult to solve the continuous optimization problem $\mathcal{P}$ :

$$
\min _{x_{1}, \ldots, x_{n} \in[0,1]} g_{G}\left(x_{1}, \ldots, x_{n}\right) .
$$

However, if $\left(x_{1}, \ldots, x_{n}\right)$ is the solution of any approximation method for $\mathcal{P}$, then (see Theorem 2 ) we can easily find a dominating set of $G$ of cardinality at most $g_{G}\left(x_{1}, \ldots, x_{n}\right)$. 
Theorem 2. Given a graph $G \in \Gamma$ on $V=\{1, \ldots, n\}$ with maximum degree $\Delta, x_{1}, \ldots, x_{n} \in[0,1]$, there is an $O\left(\Delta^{4} n\right)$-algorithm finding a dominating set $D$ of $G$ with $|D| \leq g_{G}\left(x_{1}, \ldots, x_{n}\right)$.

\section{Proofs}

Proof of Theorem 1. For events $A$ and $B$ and for a random variable $Z$ of an arbitrary random space, $P(A), P(A \mid B)$, and $E(Z)$ denote the probability of $A$, the conditional probability of $A$ given $B$, and the expectation of $Z$, respectively. Let $\bar{A}$ be the complementary event of $A$. We will use the well-known facts that $P(B) P(A \mid B)=P(A \cap B)=P(B)-P(\bar{A} \cap B)=$ $P(B)(1-P(\bar{A} \mid B))$ and $E\left(\left|S^{\prime}\right|\right)=\sum_{s \in S} P\left(s \in S^{\prime}\right)$ for a random subset $S^{\prime}$ of a given finite set $S . I \subset V$ is an independent set if $N(i) \cap I=\emptyset$ for all $i \in I$. Consider fixed $x_{1}, \ldots, x_{n} \in[0,1] . X \subseteq V$ is formed by random and independent choice of $i \in V$, where $P(i \in X)=x_{i}$. Let $X^{\prime}=\{i \in X \mid N(i) \subseteq X\}$, $X^{\prime \prime}=\left\{i \in X^{\prime} \mid N(i) \cap\left(X \backslash X^{\prime}\right) \neq \emptyset\right\}, Y=\{i \in V \mid i \notin X \wedge N(i) \cap X=\emptyset\}$, $Y^{\prime}=\{i \in Y \mid N(i) \cap Y \neq \emptyset\}$, and $I$ be a maximum independent set of the subgraph of $G$ induced by $Y^{\prime}$.

Lemma 3. $\left(X \backslash X^{\prime \prime}\right) \cup(Y \backslash I)$ is a dominating set of $G$.

Proof. Obviously, $X^{\prime \prime} \subseteq X^{\prime} \subseteq X$ and $\left(X \backslash X^{\prime}\right) \subseteq\left(X \backslash X^{\prime \prime}\right)$. If $i \in V \backslash(X \cup Y)$ then $N(i) \cap\left(X \backslash X^{\prime}\right) \neq \emptyset$, if $i \in X^{\prime \prime}$ then again $N(i) \cap\left(X \backslash X^{\prime}\right) \neq \emptyset$, and if $i \in I$ then $N(i) \cap(Y \backslash I) \neq \emptyset$.

Lemma 4. $\gamma \leq E(|X|)-E\left(\left|X^{\prime \prime}\right|\right)+E(|Y|)-E(|I|)$.

Proof. Let $D$ be a random dominating set of $G$. Because of the property of the expectation to be an average value we have $\gamma \leq E(|D|)$. With Lemma 3 and linearity of the expectation, $\gamma \leq E\left(\left|\left(X \backslash X^{\prime \prime}\right) \cup(Y \backslash I)\right|\right)=E\left(|X|-\left|X^{\prime \prime}\right|+\right.$ $|Y|-|I|)=E(|X|)-E\left(\left|X^{\prime \prime}\right|\right)+E(|Y|)-E(|I|)$ since $\left(X \backslash X^{\prime \prime}\right) \cap(Y \backslash I)=\emptyset$.

Lemma 5. $E(|X|)=\sum_{i \in V} x_{i}, E\left(\left|X^{\prime \prime}\right|\right)=\sum_{i \in V} x_{i}\left(\prod_{j \in N(i)} x_{j}\right)\left(1-\prod_{k \in N_{2}(i)} x_{k}\right)$,

$$
\begin{aligned}
& E(|Y|)=\sum_{i \in V}\left(1-x_{i}\right) \prod_{j \in N(i)}\left(1-x_{j}\right), \text { and } \\
& E(|I|) \geq \sum_{i \in V} \frac{1}{1+d_{i}}\left(1-x_{i}\right)\left(\prod_{j \in N(i)}\left(1-x_{j}\right)\right)\left(\prod_{k \in N_{2}(i)}\left(1-x_{k}\right)\right) .
\end{aligned}
$$


Proof. $E(|X|)=\sum_{i \in V} P(i \in X)=\sum_{i \in V} x_{i}$.

$$
\begin{aligned}
& E\left(\left|X^{\prime \prime}\right|\right)=\sum_{i \in V} P\left(i \in X^{\prime \prime}\right)=\sum_{i \in V} P\left(i \in X \wedge N(i) \subseteq X \wedge N(i) \cap\left(X \backslash X^{\prime}\right) \neq \emptyset\right) \\
& =\sum_{i \in V} P(i \in X) P(N(i) \subseteq X) P\left(N(i) \cap\left(X \backslash X^{\prime}\right) \neq \emptyset \mid i \in X \wedge N(i) \subseteq X\right) \\
& =\sum_{i \in V} x_{i}\left(\prod_{j \in N(i)} x_{j}\right)\left(1-P\left(N(i) \subseteq X^{\prime} \mid i \in X \wedge N(i) \subseteq X\right)\right) \\
& =\sum_{i \in V} x_{i}\left(\prod_{j \in N(i)} x_{j}\right)\left(1-P\left(N_{2}(i) \subseteq X\right)\right)=\sum_{i \in V} x_{i}\left(\prod_{j \in N(i)} x_{j}\right)\left(1-\prod_{k \in N_{2}(i)} x_{k}\right) . \\
& E(|Y|)=\sum_{i \in V} P(i \in Y)=\sum_{i \in V} P(i \notin X) P(N(i) \cap X=\emptyset) \\
& =\sum_{i \in V}\left(1-x_{i}\right) \prod_{j \in N(i)}\left(1-x_{j}\right) .
\end{aligned}
$$

A lower bound on $|I|$ (see $[1,9,2,6]$ ) is given by the following inequality $|I| \geq \sum_{i \in Y^{\prime}} \frac{1}{1+d_{i}}$. For $i \in V(G)$ define the random variable $Z_{i}$ with $Z_{i}=\frac{1}{1+d_{i}}$ if $i \in Y^{\prime}$ and $Z_{i}=0$ if $i \notin Y^{\prime}$. Hence,

$$
\begin{aligned}
& E(|I|) \geq E\left(\sum_{i \in V} Z_{i}\right)=\sum_{i \in V} E\left(Z_{i}\right)=\sum_{i \in V} \frac{1}{1+d_{i}} P\left(i \in Y^{\prime}\right) \\
& =\sum_{i \in V} \frac{1}{1+d_{i}} P(i \notin X \wedge N(i) \cap X=\emptyset \wedge N(i) \cap Y \neq \emptyset) .
\end{aligned}
$$

Because $d_{i} \geq 1, N_{2}(i) \cap X=\emptyset$ implies $N(i) \cap Y \neq \emptyset$. Hence,

$$
\begin{aligned}
& E(|I|) \geq \sum_{i \in V} \frac{1}{1+d_{i}} P\left(i \notin X \wedge N(i) \cap X=\emptyset \wedge N_{2}(i) \cap X=\emptyset\right) \\
& =\sum_{i \in V} \frac{1}{1+d_{i}} P(i \notin X) P(N(i) \cap X=\emptyset) P\left(N_{2}(i) \cap X=\emptyset\right) \\
& =\sum_{i \in V} \frac{1}{1+d_{i}}\left(1-x_{i}\right)\left(\prod_{j \in N(i)}\left(1-x_{j}\right)\right)\left(\prod_{k \in N_{2}(i)}\left(1-x_{k}\right)\right) .
\end{aligned}
$$


From Lemma 4 and Lemma 5 we have $\gamma \leq g_{G}\left(x_{1}, \ldots, x_{n}\right) \leq f_{G}\left(x_{1}, \ldots, x_{n}\right)$. Let $D^{*}$ be a minimum dominating set of $G$ and let $y_{i}=1$ if $i \in D^{*}$ and $y_{i}=0$ if $i \notin D^{*}$. Then $y_{i} \prod_{j \in N(i)} y_{j}=0$ and $\left(1-y_{i}\right) \prod_{j \in N(i)}\left(1-y_{j}\right)=0$ for every $i \in V, \gamma=\left|D^{*}\right|=\sum_{i \in V} y_{i}=g_{G}\left(y_{1}, \ldots, y_{n}\right)=f_{G}\left(y_{1}, \ldots, y_{n}\right)$, and the proof of Theorem 1 is complete.

Proof of Theorem 2. Given a graph $H$ on $n_{H}$ vertices with $m_{H}$ edges, there is an $O\left(n_{H}+m_{H}\right)$-algorithm $\mathcal{A}$ finding an independent set of $H$ with cardinality at least $\sum_{y \in V(H)} \frac{1}{1+d_{H}(y)}$, where $d_{H}(y)$ is the degree of $y \in V(H)$ in $H$ (see [2]).

First we present an algorithm that constructs a set $D \subseteq V$.

\section{Algorithm}

INPUT: a graph $G \in \Gamma$ on $V=\{1, \ldots, n\}, x_{1}, \ldots, x_{n} \in[0,1]$

OUTPUT: $D$

(1) For $l=1, \ldots, n$ do if $\frac{\partial g_{G}\left(x_{1}, \ldots, x_{n}\right)}{\partial x_{l}} \geq 0$ then $x_{l}:=0$ else $x_{l}:=1$.

(2) $X:=\left\{l \in\{1, \ldots, n\} \mid x_{l}=1\right\}$. Calculate $X^{\prime \prime}, Y, Y^{\prime}$, and $I$ using $\mathcal{A}$.

(3) $D:=\left(X \backslash X^{\prime \prime}\right) \cup(Y \backslash I)$.

END

Let $g^{*}=g_{G}\left(x_{1}, \ldots, x_{n}\right)$, where $\left(x_{1}, \ldots, x_{n}\right)$ is the input vector. Note that the function $g_{G}$ is linear in each variable. Thus, in step (1), for fixed $x_{1}, \ldots, x_{l-1}, x_{l+1}, \ldots, x_{n}$ we always choose $x_{l}$ in such a way that the value of $g_{G}\left(x_{1}, \ldots, x_{n}\right)$ is not increased. Hence, $x_{l} \in\{0,1\}$ for $l=1, \ldots, n$ and $g_{G}\left(x_{1}, \ldots, x_{n}\right) \leq g^{*}$ after step (1) of the algorithm. With Lemma $3, D$ is a dominating set, and with $|S|=E(|S|)$ for a deterministic set $S$ and Lemma $5,|D| \leq g^{*}$. It is easy to see that $\frac{\partial g_{G}\left(x_{1}, \ldots, x_{n}\right)}{\partial x_{l}}$ can be calculated in $O\left(\Delta^{4}\right)$ time. Since $G$ has $O(\Delta n)$ edges, the algorithm runs in $O\left(\Delta^{4} n\right)$ time.

\section{References}

[1] Y. Caro, New results on the independence number (Technical Report, Tel-Aviv University, 1979).

[2] Y. Caro and Zs. Tuza, Improved lower bounds on $k$-independence, J. Graph Theory 15 (1991) 99-107.

[3] R. Diestel, Graph Theory, Graduate Texts in Mathematics (Springer, 1997).

[4] N. Alon, J.H. Spencer and P. Erdös, The Probabilistic Method (John Wiley and Sons, Inc. 1992), page 6. 
[5] M.R. Garey and D.S. Johnson, Computers and Intractability, A Guide to the Theory of NP-Completeness (W.H. Freeman and Company, San Francisco, 1979).

[6] J. Harant, Some news about the independence number of a graph, Discuss. Math. Graph Theory 20 (2000) 71-79.

[7] J. Harant, A. Pruchnewski and M. Voigt, On dominating sets and independent sets of graphs, Combinatorics, Probability and Computing 8 (1999) 547-553.

[8] T.W. Haynes, S.T. Hedetniemi and P.J. Slater, Fundamentals of Domination in Graphs (Marcel Dekker, Inc., New York, N.Y., 1998), page 52.

[9] V.K. Wei, A lower bound on the stability number of a simple graph (Bell Laboratories Technical Memorandum 81-11217-9, Murray Hill, NJ, 1981).

Received 23 September 2003 Revised 15 June 2004 